\title{
ANIMALITÉ ET VÉGÉTALITÉ DANS LES REPRÉSENTATIONS DE L'HÉRÉDTÉ
}

Il est des circonstances où la science est spontanément vulgaire. Entendons par là : des circonstances dans lesquelles la connaissance que produisent les savants se distingue à peine de celle qu'ils diffusent à l'intention d'un large public éclairé. Dans de tels cas, pour reprendre une belle suggestion de 'Baudoin Jurdant ', il n'est point besoin d'épistémologie comme médiation entre la science savante et la science vulgarisée. C'est sous cet angle insolite que nous proposerons quelques réflexions sur l'histoire des représentations de l'hérédité dans une période allant de 1860 à 1960.

Pour nous être, en d'autres circonstances, longuement étendu sur certains aspects "durs " de cette histoire, nous n'aurons pas de scrupule à prendre ici quelque distance par rapport à ce redoutable champ de positivité biologique, et à l'envisager dans quelques aspects de sa "vulgarité " - ou popularité - philosophique.

L'hypothèse que nous soutiendrons se résume en peu de mots. Depuis le milieu du xix ${ }^{e}$ siècle, et jusqu'en 1960 au moins, l'hérédité n'a cessé de se confirmer comme l'un des problèmes centraux, et sans doute comme le problème théorique central, de la biologie moderne. Aussi est-il compréhensible que, par-delà les vicissitudes et les succès de la science empirique de l'hérédité, la notion ait suscité toutes sortes de réflexions générales sur la nature même de la vie. Le concept d'hérédité s'est cherché dans une série de philosophèmes qui rappellent étrangement la trilogie aristotélicienne de l'âme. On peut en effet assez aisément repérer des représentations végétalisantes, animalisantes et intellectives de l'hérédité. L'objet de cette communication est de spécifier historiquement cette assertion, et d'en proposer une interprétation philosophique.

1. Baudoin JURDANT, « Les pratiques de vulgarisation scientifique en France, GrandeBretagne et Etats-Unis", conférence donnée dans le cadre du colloque Styles scientifiques et cultures locales, C.N.R.S. et Cité des sciences et de l'industrie, 17-18 mai 1990.

Revue de synthèse : IV S. Nos 3-4, juil.-déc. 1992. 
Il serait abusif de dire que la notion biologique de l'hérédité est apparue au $\mathrm{XIX}^{\mathrm{e}}$ siècle. Mais il est historiquement justifié d'affirmer que c'est Darwin qui, récupérant un mot confiné dans la pratique des éleveurs, des vétérinaires et des psychiatres, l'a projeté sur le devant de la scène biologique, et lui a conféré le sens d'une interrogation théorique urgente et centrale. Les deux principes fondamentaux de l'Origine des espèces, à savoir le principe de "descendance avec modification" et celui de " sélection" (naturelle, sexuelle ou artificielle) présupposent tous deux, et très explicitement, un principe universel de l'hérédité des variations. Il est arrivé par exemple à Darwin d'utiliser l'expression d'" hérédité avec modification " comme synonyme de $"$ descendance avec modification $"$. Quant au principe de sélection, il ne se conçoit par définition que pour des variations " héréditaires " ${ }^{3}$. Cependant, Darwin prend bien soin, en 1859 , de ne développer aucune hypothèse sur la nature intime des phénomènes d'hérédité. Confiant dans un vieux précepte d'inspiration newtonienne, il est persuadé que l'hérédité, à la manière des forces de la Mécanique, peut être une vera causa dont la réalité se manifeste dans ses effets, sans qu'il soit besoin de spéculer sur son mécanisme ${ }^{4}$.

$\mathrm{Au}$-delà de 1859 néanmoins, ce sera une urgence théorique pour une majorité de biologistes que de spéculer sur la nature de l'hérédité. Or, deux grandes conceptions antithétiques se mettent en place dès les années 1860 , toutes deux végétalisantes, mais en un sens très différent.

La première conception est illustree par Claude Bernard, et consiste à résorber les faits d'hérédité dans ceux de nutrition. Claude Bernard n'a effectué aucun travail sur les faits d'hérédité, mais il s'est exprimé sur eux dans un contexte très particulier, celui d'un rapport rédigé en 1867 à la demande du ministre de l'Instruction publique de l'époque, Victor Duruy ${ }^{5}$, et publié sous le titre de Rapport sur les progrès et la marche de

2. Cf. Charles Darwin, On the Origin of Species, $1^{\text {re }}$ éd., Londres, John Murray, 1859, p. 350 : «On this principle of inheritance with modification, we can understand how it is that sections of genera, whole genera, and even families are confined to the same areas... "

3. Cf. Ibid., Introduction, p. 4, et chap. Iv, "Natural Selection », $\S$ « Summary of Chapter ", p. 127.

4. Pour un développement circonstancié de ces affirmations, cf. Jean Gayon, Darwin et l'après-Darwin : une histoire du concept de sélection dans la pensée évolutionniste, Paris, Kimé, 1992, chap. I.

5. Claude BERnARD, Rapport sur les progrès et la marche de la physiologie générale en France, Paris, Imprimerie impériale, 1867. Ce rapport a été réédité sans modification sous le 
la physiologie générale en France. Conformément à un souhait exprimé par le ministre, le rapport décrivait non "l'état " mais les perspectives de "progrès " des diverses branches de la physiologie. Le texte avait donc valeur programmatique, et a été l'un des ouvrages de philosophie biologique les plus lus par les biologistes français de la fin du $\mathrm{XIX}^{\mathrm{e}}$ siècle. Il contient un développement sur l'hérédité, situé dans un chapitre qui traite simultanément des phénomènes de "nutrition ", mais aussi de "génération " et d' " évolution", génération et évolution n'étant pour Claude Bernard qu'une expansion de la nutrition dans le temps ${ }^{6}$. Dans ce contexte, le physiologiste définit un programme de recherche très explicite pour tout expérimentateur qui voudrait avancer dans la compréhension de l'hérédité : il conviendra de déterminer comment les conditions nutritives assignées à l'embryon sont susceptibles de modifier simultanément "la direction des phénomènes de développement" et "l'hérédité », que Claude Bernard appelle aussi « tradition organique "

Ce programme de recherche a bien entendu des racines très anciennes. Il s'inscrit dans une philosophie biologique entretenue de manière ininterrompue depuis Aristote, et consistant à poser que nutrition et génération relèvent d'une même faculté, la faculté végétative commune à tous les êtres vivants ${ }^{8}$. Mais par-delà cette antique filiation, la représentation de

titre De la physiologie générale, Paris, Hachette, 1872. Pour une présentation plus détaillée des conceptions bernardiennes en matière d'hérédité, nous renvoyons à notre étude " Un objet singulier dans la philosophie biologique bernardienne : l'hérédité ", in La Nécessité de Claude Bernard, sous la dir. de Jacques Micheц, Paris, Méridiens Klincksieck, 1991, p. 169182.

6. Cf. C. Bernard, op. cit. supra n. 5, 1872, p. 130 : " La nutrition n'est que la génération continuée. Dans les Lecons sur les phénomènes de la vie communs aux animaux et aux végétaux, Paris, J. B. Baillière \& Fils, 1885, vol. I, Appendice, p. 387, cette idée est confirmée de la manière la plus claire : «il est impossible de séparer la nutrition de l'accroissement, du développement et de la succession des âges, c'est-à-dire de l'évolution. L'évolution c'est l'ensemble constant de ces alternatives de la nutrition; c'est la nutrition considérée dans sa réalité, embrassée d'un coup d'œil à travers le temps » (souligné par l'auteur).

7. C. BERNARD, op. cit. supra n. 5,1872 , p. 157-161.

8. Un examen attentif du corpus aristotélicien montrerait que le point de vue nutritif est précisément ce qui permet au stagyrite de distinguer les phénomènes d'hérédité de ceux de génération. Refusant le concept hippocratique de la pangenèse, Aristote ne peut expliquer la ressemblance variable de l'enfant avec le père et avec la mère par l'idée d'une double semence, constituée à partir des divers organes des deux parents. La théorie hylémorphique de la génération risque au contraire de rendre inintelligible le fait que l'enfant ressemble à la fois au père et à la mère. Or c'est précisément ici qu'intervient la conception nutritive de la génération. La semence n'est pas une empreinte des organes, mais une sorte de condensé (un " résidu ") de ce qui nourrit les organes. Cette conception, associée au principe selon lequel le sang menstruel féminin n'est pas à proprement parler dépourvu de forme, mais contient aussi un "résidu " nutritif capable d'entrer en concurrence avec le "résidu " du sperme mâle, permet à Aristote de rendre compte des faits de ressemblance avec les deux parents, aussi bien que des différences entre les enfants. ARISTOTE, De la génération des animaux, tout particulièrement IV. 3, et l'excellente analyse de Nicolas RusseL,, Like engendring like, Cambridge, Cambridge University Press, 1986, chap. II. C'est à cette antique source conceptuelle 
l'hérédité dans le langage de la nutrition rappelle des propos très explicites de Lamarck ${ }^{9}$, et est devenue, à partir de la fin du XIX ${ }^{\mathrm{e}}$ siècle, le leitmotiv du néo-lamarckisme français : Edmond Perrier, Félix Le Dantec, Étienne Rabaud, Paul Wintrebert, et bien d'autres, n'ont cessé de répéter que l'hérédité était un fait d'assimilation, et que c'était en ce sens précis qu'il fallait entendre la doctrine lamarckienne de la «modification acquise »"

La seconde conception spéculative de l'hérédité qui se met en place dès les années 1860 est illustrée par Francis Galton, et s'est précisément élaborée comme une dénégation de la conception nutritive. La spéculation galtonienne sur l'hérédité a deux aspects complémentaires. Elle a d'abord l'aspect négatif d'une critique, comme on le voit dans les textes relatant les célèbres expériences de transfusion sanguine effectuées sur des lapins: en montrant que des lapines enceintes et transfusées engendrent des petits conformes à leur propre type, et non à celui de l'animal donneur de sang, Galton estimait qu'il jetait un discrédit fatal sur l'hypothèse darwinienne de la pangenèse, mais aussi sur la vieille idée que la génération et l'hérédité sont sous la dépendance de la nutrition. L'hérédité n'est pas, en quelque manière que ce soit, une affaire de " sang ". Par conséquent, il n'est pas vrai, contrairement au vieux dicton français, que «nurture passe nature ». La « nurture » ne corrige pas la "nature " $"$. Dans son aspect affirmatif, la spéculation galtonienne consiste par ailleurs à ériger en principe la continuité des éléments « embryonnaires » au fil des générations. Dès 1865 - vingt ans avant Weismann -, la doctrine est posée de manière assez claire :

« Nous aurons une vue à peu près correcte de l'origine de notre vie, si nous comprenons que nos propres embryons ont leur origine immédiate [have

que se rattache le lamarckisme de Claude Bernard, tandis que les spéculations tardives de Darwin sur la pangenèse font évidemment penser à l'interprétation hippocratique des mêmes faits.

9. Cf. Jean-Baptiste Monet, chevalier de Lamarck, Philosophie zoologique, Paris, Dentu, 1809 , t. I, chap. vI : « Entre des individus de même espèce, dont les uns sont continuellement bien nourris, et dans des circonstances favorables à tous leurs développements, tandis que les autres se trouvent dans des circonstances opposées, il se produit une différence dans l'état de ces individus, qui peu à peu devient très remarquable. Que d'exemples ne pourrais-je pas citer à l'égard des animaux et des végétaux, qui confirmeraient le fondement de cette considération! Or, si les circonstances, restant les mêmes, rendent habituel et constant l'état des individus mal nourris, souffrants et languissants, leur organisation intérieure est enfin modifiée et la génération entre les individus dont il est question conserve les modifications acquises et finit par donner lieu à une race très distincte... "

10. Pour une vue d'ensemble du néo-lamarckisme français, voir Revue de synthèse, $n^{\circ}$ spécial, «Les néo-Lamarckiens français », sous la dir. de Jacques RoGER, juill.-déc. 1979, et Denis Buican, Histoire de la génétique et de l'évolutionnisme en France, Paris, Presses universitaires de France, 1984.

11. Francis Galton, " Experiments in Pangenesis by breeding from Rabbits of a Pure Variety, into whose Circulation Blood taken from other Varieties had previously been lar- 
sprung immediately] dans ces embryons à partir desquels nos parents se sont eux-mêmes développés, tandis que les embryons de nos parents venaient des embryons de leurs propres parents, et ainsi de suite depuis toujours. Nous devons de la sorte considérer la nature de l'humanité, et peut-être de la totalité des êtres animés, comme un système continu, qui produit sans cesse de nouvelles branches dans toutes les directions, qui s'entrelace de diverses manières, et qui bourgeonne des vies séparées à chaque point d'entrelacement ${ }^{12}$.

Ce passage, extrait du tout premier texte de Galton sur l'hérédité (en l'occurrence un texte sur l'hérédité des attributs mentaux de l'homme), contient l'image que Galton ne cessera de développer par la suite : l'hérédité représentée comme une sorte de stolon qui bourgeonne sans cesse de nouveaux individus, mais suit indéfiniment son cours autonome. On a vu là une première expression - quasi métaphysique - de ce qu'August Weismann a nommé en 1883 le principe de «continuité » et d'《indépendance » de la lignée germinale ${ }^{13}$.

Cette histoire-là est assez connue. Aussi n'insisterons-nous que sur un aspect remarquable de la spéculation galtonienne. Cette spéculation s'appuie sur une métaphore végétale, dont la signification doit être soigneusement évaluée. À partir de 1875, Galton a jugé bon d'inventer un néologisme pour exprimer sa pensée : il a proposé d'appeler «stirpe » (du latin stirpes: racine) « la somme des germes qui se trouvent dans l'œuf fécondé ». Cette "stirpe», expliquait-il, contient d'une part les germes destinés à s'exprimer dans l'organisme « apparent », mais aussi d'autres voués à demeurer « latents », quoique transmis comme les premiers à la progéniture ${ }^{14}$. L'organisme apparent résulte ainsi du développement d'une fraction du matériel héréditaire qu'un individu peut transmettre à sa descendance.

Or la métaphore végétale était doublée d'une autre analogie, qui révèle la signification de la stirpe :

« Nous pouvons comparer la stirpe à une nation, et les germes qui arrivent à un entier développement aux hommes marquants qui réussissent à devenir les représentants de la nation [...] Ce ne sont point là de vaines métaphores, mais bien des analogies parfaitement exactes; elles supportent l'examen et

gely transfused ", Proceedings of the Royal Society of London, 19, 1871, p. 394-410, et « On Blood-Relationship », ibid., 20, 1872, p. 394-402.

12. ID., « Hereditary Talent and Character », Macmillan's Magazine, 12, 1865, p. 322, souligné par l'auteur.

13. August Weismann, Uber die Vererbung, Jena, Gustav Fischer, 1883, trad. franç. dans Essais sur l'hérédité et la sélection naturelle, Paris, C. Reinwald et Cie, 1892, p. 117-156.

14. F. Galton, "A Theory of Heredity ", Contemporary Review, 27, 1875, p. 80-95, trad. franç. sous le titre "Théorie de l'hérédité ", Revue scientifique, 10, 1976, p. 198-205. 
méritent d'être poursuivies, parce qu'elles donnent à nos idées sur l'hérédité la clarté qui leur est si nécessaire $"{ }^{15}$.

Galton pense donc la « stirpe », c'est-à-dire le matériau héréditaire, sur le modèle de la représentation politique. Les caractères héréditaires portés dans les cellules germinales constituant la « stirpe " sont des " représentants ", dans l'individu actuellement vivant, des divers héritages biologiques qu'il a reçus. La raison profonde de cette analogie de second degré est celle-ci : le brassage indéfiniment renouvelé des éléments héréditaires à chaque génération fait que chaque individu ne ressemble pas seulement à ses parents, mais aussi à ses ancêtres; la " stirpe » individuelle est en fait un échantillonnage partiel des " germes " de tous les ancêtres. Théorisée sous le nom d' " hérédité ancestrale ", cette notion aboutit en définitive à solidariser les notions d'hérédité et de race. L'entrelacs bizarre des métaphores végétales et politiques révèle alors sa signification philosophique fondamentale : par-delà la subtile définition opératoire de l'hérédité qu'on doit à Galton d'avoir construite (l'hérédité comme relation statistique entre les générations successives d'une population), il s'agit d'argumenter en faveur de la réalité des types raciaux; l'individu apparent n'est qu'une instance (une « représentation ») d'un « héritage » biologique qui n'est lui-même qu'un échantillonnage transitoire d'une réalité définie par le type racial.

De semblables métaphores se retrouvent chez d'innombrables auteurs de la fin du $x^{e}{ }^{e}$ siècle. Exprimées dans un vocabulaire caractéristique du tronc, de la racine, de la souche, du lignage, elles aboutissent toujours plus ou moins explicitement à résorber l'individu dans la race.

Ainsi voyons-nous comment la biologie du $\mathrm{xIX}^{e}$ siècle a cherché ses représentations de l'énigmatique « hérédité " tantôt dans un concept végétatif (la nutrition), tantôt dans une image végétale (la racine). On sait le destin de l'un et de l'autre. Le concept physiologique et nutritif de l'hérédité a égaré des générations de néo-lamarckiens. L'image de la racine a porté les pires idéologies raciales; mais c'est aussi en elle que s'est cherché le concept expérimental de l'hérédité, dans la biométrie d'abord, dans la génétique mendélienne ensuite. 
Les représentations animalisantes de l'hérédité sont plus délicates à décrire. Car si elles existent, elles enveloppent de prime abord un paradoxe : celui consistant à penser une propriété universelle des êtres vivants dans un langage approprié à certains d'entre eux seulement. En outre, il s'agit de traditions aux contours moins définis, et plus subtilement réparties entre des discours de grande diffusion et d'autres confinés dans les secteurs les plus " durs" de la science de l'hérédité. Cette réserve étant faite, nous repérons deux grandes lignes thématiques : l'hérédité comme "mémoire " et l'hérédité comme " comportement».

\section{a) Les "théories mnémoniques de la vie».}

Il s'agit de conceptions qui ont eu une immense popularite dans les années 1870 à 1930, sous ce qualificatif même. La représentation de l'hérédité comme "mémoire " n'a en soi rien de très étonnant et n'était pas nouvelle. Elle figure par exemple très explicitement chez Maupertuis ${ }^{16}$, dans un contexte où la mémoire n'est que l'un des attributs psychiques attribués aux particules génératives des organismes, à côté de la perception, de l'intelligence et du désir. Ce qui est spécifique des « théories mnémoniques de la vie ", c'est une argumentation visant à montrer que l'analogie entre mémoire et hérédité est réelle et fondamentale, en ce sens que la mémoire est authentiquement $l a$ " fonction universelle de la matière organisée ". C'était là d'ailleurs le titre même d'un célèbre texte d'Ewald Hering, publié en allemand en 1870, puis traduit en anglais en 1880 par Samuel Butler dans la Mémoire inconsciente ${ }^{17}$. Dans le sillage de ce texte s'est constituée une vaste tradition spéculative, illustrée par Ernst Haeckel (dans sa Périgenèse des plastidules) ${ }^{18}$, Samuel Butler ${ }^{19}$, l'école néo-lamarckienne américaine (Hyatt, Packard, Cope ${ }^{20}$ et, plus

16. Pierte-Louis Moreau de Maupertuls, Système de la nature, par ex. $\$ \S \mathrm{XIV}$ et XXIII.

17. Ewald Hering, "Memory as Universal Function of Organized Mater ", in Samuel Butler, Unconscious Memory, 1920, Londres, A. C. Fifield, $3^{\mathrm{e}}$ éd., 1920, p. 63-86.

18. Ernst Haeckel, Die Perigenesis des Plastidule..., Berlin, Reimer, 1876.

19. S. BUTLER, op. cit. supra, n. 17.

20. Pour une présentation de cette école, sous l'angle qui nous intéresse ici, voir Stephen Jay Gould, Ontogeny and Phylogeny, Cambridge, Harvard University Press, 1977. 
tard, par Coutagne ${ }^{21}$, Richard Semon ${ }^{22}$, Eugenio Rignàno ${ }^{23}$ et Ludwig von Bertalanffy ${ }^{24}$. Littérature passablement confuse dans ses arguments, mais unifiée par la conviction que l'essence de l'hérédité devait pouvoir être identifiée dans quelque caractéristique physique propre aux totalités vivantes. Tantôt " vibratoires ", tantôt « électriques ", ou encore " énergétiques ", les théories mnémoniques de la vie s'accordent toutes dans une conception holistique et dynamique de l'hérédité, et sont caractéristiques d'une culture qui a vu dans la notion de processus « inconscients » une possibilité d'unification conceptuelle de l'ensemble des sciences de la vie et des sciences de l'homme ${ }^{25}$. Nous ne nous attarderons pas sur le détail de ces théories, et nous nous contenterons d'en indiquer deux implications majeures lorsqu'on les envisage comme des pensées de l'hérédité.

La première est que les organismes individuels y apparaissent comme des totalités pleinement intégrées, capables d'incorporer leur expérience jusque dans leurs structures germinales, d'où il résulte qu'ils jouent, en tant qu'individus, un rôle actif et responsable dans l'évolution de leur espèce. C'est pourquoi les théories mnémoniques de la vie ont constitué l'ossature spéculative de l'une des deux grandes modalités du néolamarckisme, celle couramment désignée sous le nom de "psycholamarckisme ». Pour les psycho-lamarckiens (cf. supra notes 17 à 24), le caractère crucial du concept d'hérédité pour la théorie biologique consistait en ce que l'hérédité n'est pas seulement une fonction de transmission passive, mais une fonction de mémorisation. L'autre grande modalité du lamarckisme a consisté dans l'interprétation physio-mécaniste de l'hérédité comme phénomène d'assimilation nutritive. Le psycho-lamarckisme a été plus virulent dans les pays de langue allemande et anglaise, le physio-mécanisme étant plus répandu dans les pays de langue latine, en particulier en France et en Italie. Toutefois, la ligne de partage entre les conceptions «mnémonique » et « nutritive » de l'hérédité a souvent été

21. George Coutagne, Recherches expérimentales sur l'hérédité chez les vers à soie, thèse, Université de Paris, 1902, publ. dans Bulletin scientifique de la France et de la Belgique, 37, 1903. Coutagne admet avec Weismann qu'il y a dans chaque cellule générale des tendances héréditaires distinctes pour chaque organe du futur soma. Il les appelle « mnémons », et.soutient qu'ils entrent en lutte au moment de la fécondation.

22. Richard Semon, Die Mneme..., $3^{e}$ éd., Leipzig, Engelmann, 191 1, Das Problem der Vererbung erworbener Eigenschaften, Leipzig, Engelmann, 1912.

23. Eugenio Rignano, La Mémoire biologique, Paris, Alcan, 1923 ; Qu'est-ce que la vie?, Paris, Alcan, 1927.

24. Ludwig von BertalanfFy, « Eine mnemonische Lebenstheorie als Mittelweg ewischen Mechanismus und Vitalismus ", Biologia generalis, 1927; "L'état actuel du problème de l'évolution ", Scientia, 46, suppl. 27, 1929, p. 37-47 et 79-88.

25. Freud à cet égard n'est qu'un avatar, mais sans doute le seul qui ait eu de l'avenir dans ce créneau idéologique encombré. 
assez floue. Les deux métaphores, végétale et animale, se prêtaient toutes deux avec une égale efficacité à la popularisation de l'idée d'hérédité comme modification individuellement acquise ${ }^{26}$.

La seconde implication du concept de l'hérédité comme mémoire se reconnaìt dans un intérêt fondamental pour les phénomènes de récapitulation, envisagés comme une confirmation massive, à l'échelle géologique, de la conception mnémonique de la vie. Comme l'a justement noté Stephen Jay Gould, récapitulation et mémoire se prêtaient à une forte analogie dans la mesure où toutes deux se laissent décrire comme un processus indéfini d'accumulation par condensation et addition ${ }^{27}$. C'est pourquoi l'on a parfois remarqué que la théorie de la récapitulation avait occupé dans la théorie biologique de la fin du $\mathrm{xIx}^{e}$ siècle une place investie plus tard par la génétique ${ }^{28}$.

\section{b) Conceptions comportementales de l'hérédité.}

Ce sont des réinterprétations des précédentes dans un langage de l'action et dans une forme philosophique où l'on reconnaît en général assez clairement la marque de la Naturphilosophie. Mais on ne saurait, cette fois, identifier une tradition théorique dûment située et étiquetée.

D'Arcy Wentworth Thompson, dans son légendaire ouvrage sur $\mathbf{L a}$ Croissance et la forme (1917), a très fermememt exprimé l'engagement philosophique caractéristique de toutes les conceptualisations dynamiques $^{29}$ de l'hérédité. En toute rigueur, il serait philosophiquement plus exact de parler de conceptualisations énergétistes de l'hérédité. Mais l'on sait comment la physique de l'énergie a, de Leibniz à Mach, contribué à rendre les deux termes quasiment synonymes. Quoi qu'il en soit, d'Arcy Thompson récuse toute représentation de l'hérédité comme substance. C'est pour lui une illusion de croire que la transmission des attributs héréditaires pourrait être exhaustivement comprise comme continuité généalogique d'une entité substantielle quelconque, que ce soit la «cel-

26. La distinction ne doit pas être exagérée ; l'usage assez universel du concept d'assimilation dans les deux traditions montre que beaucoup d'auteurs y voyaient un dépassement du mécanisme et du vitalisme. Voir, par ex., E. Rignano, op. cit. supra n. 23.

27. S. J. Gould, op. cit. supra n. 20, p. 99. Sur l'histoire des théories mnémoniques de la vie, on consultera aussi avec fruit Peter J. Bowler, The Eclipse of Darwinism : Anti-Darwinian Evolution Theories in the Decade around 1900, Baltimore, The Johns Hopkins University Press, 1983.

28. Sur ce point, et sur la littérature afférente, voir J. GAYON, « Le concept de récapitulation à l'épreuve de la théorie darwinienne de l'évolution ", in Histoire du concept de récapitulation, dir. de Paul Mengal, Paris, Masson, sous presse.

29. En toute rigueur, il serait plus exact de parler de conceptualisations énergétistes. Mais l'on sait comment la physique de l'énergie a contribué à rendre les deux termes quasiment synonymes. 
le noyau, le chromosome, le gène, ou toute autre particule que l'on voudra :

«Dans toutes les hypothèses comme la " pangenèse ", dans toutes les théories qui attribuent des propriétés spécifiques aux micelles, aux chromosomes, aux idioplastes, aux ides, ou à d'autres particules constitutives du protoplasme ou de la cellule, nous sommes sujets à tomber dans l'erreur consistant à attribuer à la matière ce qui relève de l'énergie et se manifeste comme force; ou, plus rigoureusement parlant, à attribuer à des particules matérielles individuelles ce qui est dû à l'énergie de leur collocation. [...] $\mathrm{Si}$, avec Weismann et quelques autres, nous parlons d'une "substance héréditaire ", une substance qui se sépare du corps parental et transmet à la nouvelle génération les caractéristiques de la précédente, nous ne pouvons justifier une telle manière de nous exprimer qu'en postulant que cette particule essentielle de matière est le véhicule essentiel d'une distribution particulière d'énergie, capable de produire du mouvement, ou d'accomplir un "travail". Car, comme le disait Newton, ce n'est rien dire que d'affirmer qu'une certaine chose " est dotée d'une qualité occulte spécifique" par laquelle elle produirait ses effets. [...] Les choses que nous voyons dans la cellule sont moins importantes que les actions que nous y reconnaissons $"{ }^{30}$.

Ces quelques lignes résument parfaitement l'esprit d'un courant de pensée qui a traversé les interrogations fondamentales de la science de l'hérédité tout au long du $\mathrm{xx}^{\mathrm{e}}$ siècle, en dépit de l'atmosphère atomistique dominante induite par les triomphes expérimentaux du paradigme mendélien.

Les conceptions dynamiques de l'hérédité sont loin d'être restées dans le seul champ des spéculations verbales. Dans les années 1940-1950, autrement dit dans la période héroïque de la biologie moléculaire, les théoriciens ont constamment hésité entre deux interprétations de la « spécificité " structurale et fonctionnelle des protéines. L'une consistait à dire que cette spécificité dépendait totalement de «molécules maîtresses " (master molecules), capables de déterminer strictement la configuration des protéines. Ces molécules moules pouvaient en l'occurrence être soit des inducteurs externes (donc le substrat même de l'action protéique), soit des inducteurs internes (par exemple, l'ADN). L'autre interprétation consistait à penser la spécificité protéique dans un langage de "l'équilibre dynamique", la spécificité étant conçue comme un état métastable, à la confluence de divers inducteurs internes et externes. Cette hésitation théorique fondamentale, et très explicite dans de nombreux textes publiés aux alentours de 1950, a été très bien résumée en

30. D'Arcy Wentworth Thompson, On Growth and Form, nouv. éd., Cambridge, Cambridge University Press, 1942, p. 288-289 (italiques et guillemets dans le texte). 
1955 par David Nanney, au cours d'un grand symposium sur Les Bases chimiques de l'hérédité :

" Deux concepts des mécanismes génétiques ont persisté côte à côte tout au long du développement de la génétique moderne, bien que l'un de ces concepts ait été beaucoup plus fortement mis en avant [...] Le premier est connu sous le nom de concept de la " molécule maitresse". Ce concept présuppose un type spécial de matériau, distinct du reste du protoplasme, et dirigeant les activités et fonctions de la cellule à la manière d'un réservoir d'information. Dans sa forme la plus simple, ce concept situe les " molécules maîtresses " dans les chromosomes et attribue les caractéristiques d'un organisme à leur configuration spécifique ; tous les autres constituants cellulaires sont alors considérés comme relativement peu importants, sinon comme serviteurs des maîtres. C'est là l'essence de la Théorie du Gène, qui suggère un gouvernement totalitaire. [...] Le second concept du mécanisme génétique est plus difficile à formuler, et l'évidence expérimentale en sa faveur est aussi plus difficile à obtenir. Nous désignerons ce concept comme celui de "l'état stationnaire". Par ce concept de "l'état stationnaire", nous entendons une organisation dynamique, capable de se perpétuer par elle-même, et mettant en jeu un ensemble d'espèces moléculaires qui doivent leurs propriétés non aux caractéristiques de l'une quelconque des sortes de molécules, mais aux relations fonctionnelles et réciproques entre ces espèces moléculaires ${ }^{31}$.

L'on voit donc en quelle manière la science de l'hérédité ne s'est pas exclusivement tenue dans des philosophèmes végétalisants. Les représentations de l'hérédité comme "mémoire", et plus subtilement, comme comportement d'un système dynamique, relèvent de philosophèmes animalisants. Nous aurions pu, bien sûr, évoquer des réflexions beaucoup plus explicitement orientés dans une telle direction. Les spéculations des psychologues sur l'hérédité (Baldwin, Lloyd Morgan, Piaget) eussent été à cet égard particulièrement suggestives, en ce qu'elles ont précisément consisté à questionner les concepts des généticiens au nom de la notion de comportement ${ }^{32}$. Toutefois, il nous a semblé plus éclairant de chercher des indices dans le discours biologique orthodoxe.

Il convient, enfin, de prévenir une objection que l'on pourrait faire à l'analyse que nous venons de développer. Si, en effet, les conceptions

31. David L. NANNEY, "The Role of Cytoplasm in Heredity", in A Symposium on the Chemical Basis of Heredity, 1956, William D. McElror \& Bentley Glass, eds, Baltimore, The Johns Hopkins Press, 1957, p. 134-135. Pour une illustration exemplaire de l'alternative résumée par Nanney, voir Jacques Monod, "The Phenomenon of Enzymatic Adaptation ", Growth Symposium, II, 1947, p. 223-289.

32. L'entreprise est particulièrement évidente dans le cas des réflexions de Jean Piaget sur la " phénocopie ». Voir, en part., les "Conclusions générales " de Adaptation vitale et psychologie de l'intelligence, Paris, Hermann, 1974, p. 103-109. 
" mnémoniques » de l'hérédité sont très intuitivement animalisantes dans leur contenu, et "populaires » dans leur diffusion, il n'en va pas de même pour les conceptions «dynamiques », qui ne sont ni immédiatement intuitives ni populaires. Le caractère animalisant des représentations dynamiques de l'hérédité peut toutefois être exprimé de manière assez simple, pour peu que l'on mobilise des catégories philosophiques traditionnelles. Voir, en effet, l'hérédité comme l'état physiologique d'un système, plutôt que comme une information atomisée, c'est invoquer une philosophie biologique fondée sur le refus de réduire la forme à la matière, ou l'acte à la puissance. En d'autres termes, c'est invoquer une vision aristotélicienne de la nature de la vie, dans laquelle la spécificité de l'organisation vitale est comprise comme l'acte indéfiniment continué d'une psyché, une âme, qui imprime son activité organisatrice à une diversité matérielle indéterminée. C'est en ce sens philosophique que les conceptions de l'hérédité comme « organisation dynamique, capable de se perpétuer elle-même " nous semblent relever d'une intuition animiste des phénomènes les plus fondamentaux de la vie. De telles conceptions ne sont sans doute pas naivement populaires, comme le sont les conceptions mnémoniques de l'hérédité, mais elles illustrent un régime spéculatif du discours scientifique dans lequel la "positivité » est quasiment indistinguable de la catégorisation philosophique traditionnelle. Les conceptions «dynamiques » de l'hérédité ressortissent donc à ce que l'on pourrait appeler une vulgarité philosophique spontanée, et sans doute inévitable, de la science. Elles ont en fait constitué l'élaboration philosophique ésotérique de l'identification de la vie et de la mémoire. Un tel régime de discours scientifique, tout à la fois populaire et philosophiquement hermétique, est courant dans l'histoire des sciences, lorsqu'un concept ne trouve pas à se construire comme une hypothèse opératoirement éprouvable. Il n'y a pas alors place pour une vulgarisation didactique ordinaire du savoir positif. Il y a plutôt complicité entre métaphore naïve et concept hermétique.

CONCEPTIONS DIANOETRUES DE L'HEREDITE : LA PROBlématique linguistique de la GÉNETtique MOlÉCulaire

Comme on sait, le débat que nous venons d'évoquer s'est soldé en génétique moléculaire par une victoire à peu près complète de l'interprétation de l'hérédité comme propriété générique des «master molecules " (molécules maîtresses). Mais dans cette victoire même, on peut 
reconnaitre le dernier avatar de la parabole aristotélicienne qui fait l'objet de la présente communication.

L'histoire est bien connue. Les dogmes fondamentaux de la génétique moléculaire se sont exprimés dans un vocabulaire profondément marqué par des analogies linguistiques. Il est à peine besoin de rappeler celles-ci, tant elles sont passées dans la culture scientifique ordinaire. "Linéarité du message chromosomique ", " codage ", " double articulation", "ponctuation", "phase de lecture" des acides nucléiques, " expression" des gènes, "transcription" de l'ADN en ARN, ARN "messager », « traduction " de celui-ci en séquence peptidique, interprétation de la régulation génétique en termes de "signaux " : toutes ces expressions désignent autant de dogmes fondateurs, et indiquent l'insistance avec laquelle les généticiens ont recouru à des concepts de nature linguistique.

Il est intéressant de s'interroger sur le statut de ces analogies, fondatrices d'un nouveau concept de l'hérédité. Or, contrairement à une idée répandue, ces analogies n'ont pas été de simples commodités d'expression, un tribut en quelque sorte ordinaire à l'anthropomorphisme de tout langage scientifique. L'historien constate en effet que, du milieu des années 1950 à 1970 environ (c'est-à-dire jusqu'aux débuts du " génie génétique »), de nombreux biologistes, soutenus par d'illustres linguistes et anthropologues, ont soutenu que l'analogie entre la structure des langues humaines et celle des messages génétiques était réelle ${ }^{33}$. Ainsi at-on fait valoir qu'il y a un authentique isomorphisme entre la double articulation caractéristique des langues humaines naturelles et la hiérarchie structurale des messages encodés dans l'ADN. De la même manière, en effet, que les messages parlés des hommes se distinguent de tous les autres modes d'expression en ce qu'ils ne sont en aucune façon des idéogrammes, les filaments d'ADN se distinguent des autres molécules biologiques et, en définitive, de toutes les structures biologiques, en ce qu'elles sont des molécules dont la fonction primaire est déterminée, non par la configuration tridimensionnelle de la molécule, mais par la structure linéaire de la séquence nucléotidique (la configuration tridimensionnelle de la double hélice d'ADN et de toute la machinerie associée, étant en quelque sorte mise au service de la fonction primaire de duplication et de transcodage de l'information). Comme l'a fait remarquer le linguiste Roman Jakobson, le caractère le plus troublant de l'analogie est que la " double articulation » constitue l'un des critères opératoires les plus sûrs

33. On trouvera une récapitulation suggestive de ce genre de littérature in Roman JAKOBson, " Relations entre la science du langage et les autres sciences », in Essais de linguistique générale, Paris, Minuit, 1973, vol. 2, p. 9-76. Le texte le plus significatif est peut-être le manifeste co-signé en 1968 par François JACOB, Claude Lévi-Strauss, Philippe L'Hermer, Roman JAKoBson, sous le titre "Vivre et parler », Lettres françaises, févr. 1968, p. 1221-1222. 
pour distinguer les langues naturelles des autres systèmes de communication, artificiels ou animaux. Il est tout à fait remarquable que la plus puissante des analogies que l'on puisse faire valoir entre phénomènes vitaux et phénomènes de culture joue entre ce qu'il $\mathrm{y}$ a de plus universel et de plus élémentaire dans la vie, d'une part, et ce qu'il y a de plus distinctif dans le monde symbolique de l'homme, d'autre part. La prégnance du parallélisme est en tout cas assez forte pour que l'on puisse reconnaître dans les dogmes fondamentaux de la génétique moléculaire quelque chose comme une représentation dianoétique de l'hérédité.

Cela dit, l'analogie prête flanc à des réserves, que les fondateurs de la biologie moléculaire ont été les premiers à relever. Ainsi Jacques Monod a-t-il très tôt fait observer que si la séquence nucléotidique est un " message ", ce message est non réversible, et se comporte comme un " ordre ", au sens cybernétique du terme. Si donc il y a quelque chose de " linguistique " dans le comportement des molécules héréditaires, ce linguistique n'a rien à voir avec un fait de communication. De même Francis Crick, impliqué au premier chef dans la découverte du code génétique, aimait à répéter qu'en toute rigueur, le généticien ne devrait pas parler de code, mais d'" effet magnétophone" (" tape, not code ").

On pourrait relever bien d'autres critiques du même ordre ${ }^{34}$, toutes susceptibles de tempérer l'anthropomorphisme linguistique de la génétique moléculaire. Mais on constaterait bien vite que la plupart d'entre elles conduisent à substituer à l'analogie entre hérédité et langues humaines une autre analogie, entre hérédité et langage-machine des robots. Dans un beau livre de vulgarisation publié en 1956 sous le titre Logique de la vie, Albert Ducrocq ${ }^{35}$ fait remarquer que si les chaînes d'ADN sont des séquences constituées par l'enchaînement de quatre sortes de nucléotidiques, l'association des bases dans la double hélice ne peut se faire que d'une manière déterminée : à l'adénine est toujours associée une molécule de thymine, et la guanine ne s'unit qu'à la cytosine :

« Autrement dit, le ruban d'acide désoxyribonucléique nous apparaît comme une sorte d'échelle de corde en hélice dont les barreaux sont de deux types seulement [...] Telle est la grande révélation : l'histoire de la vie est écrite en langage binaire ! [...] À l'instar de la langue binaire parfaite, la suite des AT et de GC du ruban génoïde est une langue de plein emploi en $\propto$ sens que

34. Voir, par ex., les témoignages recueillis par Horace F. Judson dans The Eigth of Creation: The Makers of the Revolution in Biology, New York, Simon and Schuster, 1979, italiques dans le texte.

35. Albert Ducroce, Logique de la vie, Paris, Julliard, 1956. 
toutes les combinaisons sont possibles, une suite quelconque de ces symboles dans n'importe quel ordre voulant toujours dire quelque chose... $"{ }^{36}$.

Ce texte est très intéressant car il se situe à la charnière de deux représentations philosophiques de l'hérédité dans la science contemporaine : tantôt science du code langagier de la vie, tantôt science de la machinerie organique. Les biologistes ont souvent raisonné comme si chacun des termes de l'alternative était une manière de dénoncer la naïveté anthropomorphique de l'autre. En réalité, les deux termes sont solidaires dans une vision des êtres vivants comme des automates calculateurs; automates dont l'algorithme de construction concilie la plus grande variabilité avec la plus grande stabilité et la plus grande économie de moyens. Le philosophème est irréductiblement linguistique et technique.

CONCLUSIONS

Faut-il insister? Nous avons convié le lecteur à un parcours aristotélicien dans l'histoire des représentations philosophiques de l'hérédité élaborées par les savants. À ce parcours désinvolte, on objectera que, depuis plus d'un siècle, le prestige de la science de l'hérédité a résulté de son efficacité pragmatique plus que de ses thèmes.

Sans doute. Mais à mesure que l'hérédité s'est imposée comme l'objet central de la théorie biologique, les savants ont projeté sur cet objet des schèmes philosophiques somme toute très classiques quant à la nature de la vie. C'est ainsi que la trilogie aristotélicienne de l'âme a trouvé occasion de résurrection anonyme.

Dans le fouillis de concepts et d'images que nous avons exhumés, on observera que l'ordre chronologique n'est pas indifférent. Du végétal à l'animal, et de l'animal à l'intellectif, la succession est sans ambiguîté. Bon an mal an, les penseurs et expérimentateurs de l'hérédité ont parcouru de bas en haut la hiérarchie aristotélicienne de l'âme.

On remarque, enfin, l'omniprésence, dans tous les discours examinés de la notion d'" analogie réelle ". C'est là un signe caractéristique d'une rationalité qui ne se satisfait pas, pour le meilleur et pour le pire, de concepts définis de manière opératoire. Dans une telle rationalité, la distance entre connaissance "savante" et connaissance "vulgaire " est floue, et il faut peu de chose pour passer de la trivialité à la plus grande

36. Ibid., p. 164-165. 
sophistication. L'ensemble des conceptions de l'hérédité que nous avons examinées a un statut paradoxal : ne relevant pas du discours technique ordinaire de la science "savante", mais tout autant ininterprétable comme des vulgarisations a posteriori, elles ressortissent à ce que l'on pourrait nommer la vulgarité philosophique spontanée de la science. Considérées isolément, ces conceptions sont transparentes à tout oreille, profane ou éduquée; mais elles sont profondément opaques dans leur historicité.

Jean GAYON, Université de Bourgogne, Dijon. 